\title{
REPORTE DE CONDICIONES SISTÉMICAS DE pacientes adultos. Facultad de Odontología, UNIVERSIDAD DE ANTIOQUIA, 2011
}

\author{
Laura Cecilia Tamayo-López, odontóloga $a_{1}$, Verónica María Medina-Piedrahíta, odontóloga \\ Luisa Fernanda Pérez-Hernández, odontóloga ${ }_{1}$, María Isabel Correa-Giraldo, est. odontología ${ }_{1}$, \\ Natalia Becerra-Moreno, odontóloga $a_{1}$ Ana Lucila Vanegas-Rodríguez, odontóloga ${ }_{1}$, \\ Leonor Victoria González-Pérez, $\mathrm{MSc}_{1}$, Andrés A. Agudelo-Suárez ${ }^{\star}$ Esp., Ph.D. ${ }_{1}$
}

${ }_{1}$ Facultad de Odontología, Universidad de Antioquia, Colombia

Recibido: 13 de marzo del 2013. Aprobado: 19 de abril del 2013.

*Autor de correspondencia: Andrés Agudelo Suárez, Facultad de Odontología, Universidad de Antioquia, Medellín, Colombia, (57) 4 219 67 72, Calle 64 n. ${ }^{\circ} 52-59$, correo electrónico: oleduga@gmail.com

Cómo citar este artículo: Tamayo-López LC, Medina-Piedrahíta VM, Pérez-Hernández LF, Correa-Giraldo MI, Becerra-Moreno N, Vanegas-Rodríguez AL, González-Pérez LV, Agudelo-Suárez AA. Reporte de condiciones sistémicas de pacientes adultos. Facultad de Odontología, Universidad de Antioquia, 2011. Revista Nacional de Odontología. 2013; 9(16): 27-33.

Resumen. Introducción: el objetivo de esta investigación fue determinar la prevalencia y la concordancia en el reporte de condiciones sistémicas entre la historia clínica odontológica (HC) y la entrevista telefónica (ET), en los pacientes que asisten a dos clínicas del adulto de la Facultad de Odontología de la Universidad de Antioquia. Métodos: estudio transversal ( $\mathrm{n}=104)$. Se evaluaron las HC y se registraron las diez condiciones sistémicas más frecuentes según registros epidemiológicos de Medellín. Se realizó et para verificar información de las HC. Se calcularon prevalencias y el nivel de concordancia entre resultados de la HC y la ET mediante el índice kappa y su intervalo de confianza al $95 \%$ ( $\mathrm{IC}_{95 \%}$ ), según variables. Resultados: la condición sistémica más prevalente fue la enfermedad ocular ( $58 \%$ según ET y $30 \%$ según HC y diferencias significativas p < 0,001), y la menos prevalente fue el hipertiroidismo ( $3 \%$ según HC y 5\% según ET, sin diferencias significativas). El análisis kappa mostró que las concordancias más débiles se presentaron para el hipertiroidismo $\left(0,22 ; \mathrm{IC}_{95 \%}-0,38-0,82\right)$ y la enfermedad ocular $\left(0,37 ; \mathrm{IC}_{95 \%} 0,20-0,72\right)$. Se evidenciaron variaciones en los índices de concordancia de acuerdo con sexo, clínica y edad. Conclusiones: existen variaciones en las prevalencias reportadas por HC y por ET debidas a dificultades a la hora de diligenciar los antecedentes médicos personales, que produjeron un subregistro en la HC.

Palabras clave: anamnesis, estadística como asunto, historia clínica, prevalencia.

Reporting Systemic Conditions in Adult Patients. School of Dentistry, Universidad de Antioquia, 2011

Abstract. Introduction: The goal of this research project was to determine the prevalence and concordance of reports of a group of systemic conditions by means of Dental/Medical Records (MRs) and telephonic surveys (Ts) in patients consulting at two adults' clinics at the Faculty of Dentistry of the University of Antioquia. Methods: Cross-sectional study $(\mathrm{n}=104)$. We evaluated clinical records and registered the 10 most frequent systemic diseases according to the epidemiological data in Medellin (Colombia). We used Ts to corroborate previous information on the MRs. We measured the prevalence of the diseases and determined a concordance level between the results of MR and Ts through a kappa index with a $95 \%$ confidence interval $\left({ }_{95 \%} \mathrm{CI}\right)$; according to different variables. Results: The most prevalent systemic disease was ocular disease ( $58 \%$ Ts and 30\% MR, statistically significant $\mathrm{p}<0.001)$ and the less prevalent one was hyperthyroidism $(3 \%$ MR and $5 \% \mathrm{Ts}$, no statistically significant). Kappa analyses showed the weakest concordance in the case of hyperthyroidism $\left(0.22{ }_{95 \%} \mathrm{CI}-0.38-0.82\right)$ and in case of ocular disease ( $\left.0.37 ;{ }_{95 \%} \mathrm{Cr} 0.20-0.72\right)$. Variability in the prevalence and kappa indexes was observed related to gender, type of clinic and age interval. Conclusions: Variations in the reported prevalence between the MR and TS were found. This situation may be explained as originating in difficulties completing medical antecedents, which cause an underreporting in the MR.

Keywords: anamnesis, statistics as topic, medical history, prevalence.
Relatório de condições sistemáticas de pacientes adultos. Faculdade de Odontologia, Universidade de Antioquia, 2011

Resumo. Introdução: o objetivo desta pesquisa foi determinar a prevalência e a concordância no relatório de condições sistêmicas entre o prontuário médico odontológico e a entrevista telefônica (ET) nos pacientes que vão a duas clínicas do adulto da Faculdade de Odontologia da Universidade de Antioquia. Métodos: estudo transversal $(\mathrm{n}=104)$. Avaliaram-se os prontuários e se registraram as dez condições sistêmicas mais frequentes segundo registros epidemiológicos de Medellín. Realizou-se ET para verificar informação dos prontuários. Calcularam-se prevalências e o nível de concordância entre resultados do prontuário e da ET mediante o índice kappa e seu intervalo de confiança a $95 \%\left(\mathrm{IC}_{95 \%}\right)$, segundo variáveis. Resultados: a condição sistêmica mais prevalente foi a doença ocular (58\% segundo ET e 30\% segundo prontuários e diferenças significativas $p<0,001$ ), e a menos prevalente foi o hipertireoidismo (3\% segundo prontuários e $5 \%$ segundo ET, sem diferenças significativas). A análise kappa mostrou que as concordâncias mais fracas se apresentaram para o hipertireoidismo $\left(0,22 ; \mathrm{IC}_{95 \%}-0,38-0,82\right)$ e a doença ocular $\left(0,37 ; \mathrm{IC}_{95 \%} 0,20-0,72\right)$. Evidenciaram-se variações nos índices de concordância de acordo com sexo, clínica e idade. Conclusões: existem variações nas prevalências relatadas pelo prontuário e pela ET devidas a dificuldades na hora de diligenciar os antecedentes médicos pessoais, que produziram um sub-registro no prontuário.

Palavras-chave: anamnese, estatística como assunto, prontuário médico, prevalência. 


\section{Introducción}

Este trabajo fue aprobado como ejercicio académico en el curso de investigación del pregrado de Odontología, Facultad de Odontología, Universidad de Antioquia. El objetivo fue determinar la prevalencia y la concordancia en el reporte de condiciones sistémicas entre la historia clínica odontológica (HC) y la entrevista telefónica (ET), en los pacientes que asisten a dos clínicas del adulto de la Facultad de Odontología de la Universidad de Antioquia.

Existen pacientes con diferentes compromisos sistémicos, que por sus cambios biológicos, hormonales, efectos medicamentosos o reacciones psicológicas pueden influir o modificar el plan de tratamiento odontológico $[1,2]$. Es por ello que para la atención de estos pacientes se deben seguir protocolos específicos adaptándolos a las necesidades individuales, para incidir eficaz y eficientemente en su necesidad de salud bucal y prevenir complicaciones médicas durante el tratamiento odontológico [3, 4].

En este contexto, es fundamental generar reflexiones entre la comunidad académica para que capacite y conciencie a los estudiantes de pre y posgrado de la importancia del registro de los antecedentes médicos personales del paciente, y a la comunidad administrativa [5], para que se evalúe la calidad en el diligenciamiento de la HC, y a partir de esto construir protocolos de atención específicos para el tipo de pacientes que asisten a las clínicas de la Facultad de Odontología de la Universidad de Antioquia.

La historia clínica (HC) que actualmente se diligencia en la Facultad de Odontología de la Universidad de Antioquia está compuesta por: 1) identificación; 2) motivo de consulta; 3 ) enfermedad actual; 4) antecedentes médicos y odontológicos personales; 5) antecedentes médicos y odontológicos familiares; 6) estado socioeconómico; 7) examen físico general y 8) examen estomatológico con sus respectivos anexos. Con esta información se establecen los diagnósticos presuntivos, se registran las ayudas diagnósticas y se establecen los diagnósticos definitivos, pronósticos y plan de tratamiento.

Una revisión de la literatura científica sobre las condiciones sistémicas prevalentes en los pacientes atendidos en los servicios de odontología dentro de contextos académicos y de servicios particulares e institucionales da cuenta de cómo en Colombia y en otros países las publicaciones son escasas [6-8]. Por ello se decidió realizar este estudio encaminado a de- terminar la prevalencia y la concordancia de las enfermedades sistémicas reportadas en los pacientes que asisten a dos clínicas del adulto de pregrado y posgrado de la Facultad de Odontología de la Universidad de Antioquia.

\section{Métodos}

Se trata de un estudio transversal. Del listado total de los pacientes registrados tanto en el archivo clínico como en los libros de las auxiliares de las clínicas del adulto II (vi semestre) e integral del adulto con énfasis en periodoncia y prostodoncia (posgrado) del semestre 2010-01 de la Facultad de Odontología de la Universidad de Antioquia, se revisaron las HC correspondientes y se seleccionó una muestra por conveniencia de 104 HC para igual número de pacientes (61 de pregrado y 43 de posgrado).

En un primer momento, se revisó el ítem de antecedentes médico-personales con el fin de establecer las condiciones sistémicas de los pacientes, haciendo énfasis en las diez enfermedades más prevalentes según los estudios epidemiológicos de la ciudad de Medellín y Antioquia [9]. Las enfermedades escogidas fueron: migraña, hipertensión arterial, hipotiroidismo, asma, reacciones alérgicas, diabetes, hipertiroidismo, gastritis, enfermedad ocular y dislipidemias. La presencia de estas enfermedades (Sí/No) fue definida por el grupo investigador de acuerdo con los parámetros establecidos en la literatura, según los signos y síntomas para cada patología [1-4]. Esta información fue sistematizada en un fichero de Excel para Windows.

En una segunda fase, se aplicó una encuesta telefónica (el instrumento de recolección de información se encuentra disponible si se solicita a los autores) a los mismos pacientes de la primera fase. Dicha encuesta contenía 100 preguntas sobre signos y síntomas (diez preguntas para cada una de las enfermedades seleccionadas). Con base en las respuestas brindadas por la población entrevistada, por consenso del grupo investigador se definía la presencia o no de cada una de las enfermedades anteriormente mencionadas. Dicha información se recopiló en un fichero, al igual que los antecedentes médicos de la primera fase.

Otras variables incluidas en el estudio fueron: edad (años cumplidos), sexo y la clínica en la cual son atendidos. Todos los instrumentos fueron probados por prueba piloto con el fin de asegurar su consistencia interna. Así mismo, se realizó un análisis de con- 
cordancia entre las investigadoras (MICG y LFPH) para asegurar que los criterios de reporte de las enfermedades analizadas fueran adecuados para los objetivos del estudio. El porcentaje de acuerdo fue del $95 \%$.

Se calcularon frecuencias absolutas y relativas por cada variable de estudio; posteriormente se estimaron las prevalencias reportadas por la HC y por la ET en cada una de las patologías, segmentando por sexo, intervalo de edad ( $\leq 50$ años, $\geq 51$ años) y tipo de clínica. Por último, se realizó un análisis de concordancia entre los datos recogidos por la HC y la ET, mediante el índice Kappa, con su intervalo de confianza al 95\% $\left(\mathrm{IC}_{95 \%}\right)$, y se determinó la fuerza de concordancia dependiendo del valor del índice: $<0,20$ pobre; $0,21-0,40$ débil; $0,41-0,60$ moderada; $0,61-0,80$ buena, $0,81-1,00$ muy buena $[10,11]$.

Para el análisis de los datos se emplearon los siguientes programas: sPSs 19.0 (Chicago IL, USA) y Epidat 3.1 (Dirección Xeral de Innovación e Xestión da Saúde Pública, Xunta de Galicia, España, Organización Panamericana de la Salud e Instituto Superior de Ciencias Médicas de La Habana) y la calculadora en línea para el cálculo del índice Kappa disponible en internet (Unidad de epidemiología clínica y bioestadísticaComplexo Hospitalario Universitario A Coruña-http:// www.fisterra.com/mbe/investiga/kappa/Kappa.xls)

El estudio cumplió con los requisitos éticos de la investigación en salud (Resolución 008430 de 1993) [12], consentimiento informado para participar en el estudio, protección de la identidad de la población entrevistada, la confidencialidad y veracidad de la información. Se considera una investigación sin riesgo, puesto que no se interviene en las características físicas, psicológicas o sociales de los pacientes selecciona- dos en el estudio. Esta investigación fue aprobada como ejercicio académico en el pregrado de Odontología de seis coautoras, y, a su vez, el proyecto fue aprobado por el Comité de Bioética de la Facultad de Odontología de la Universidad de Antioquia.

\section{Resultados}

Para esta investigación, más de dos terceras partes de la población estudiada la constituyeron mujeres (72\%) y más de la mitad fue atendida en la clínica de pregrado; el 58\% de ellas, menor de 50 años, con una media de edad de 46,5 ( $\pm 12,4)$ años (tabla 1$)$.

Tabla 1. Características sociodemográficas de la población estudiada

\begin{tabular}{|l|l|c|c|}
\hline \multicolumn{2}{|c|}{ Variables } & n & $\%$ \\
\hline \multirow{4}{*}{ Sexo } & Hombre & 29 & 27,9 \\
\cline { 2 - 4 } & Mujer & 75 & 72,1 \\
\hline \multirow{4}{*}{ Edad } & $\leq 30$ & 11 & 10,6 \\
\cline { 2 - 4 } & $31-40$ & 22 & 21,2 \\
\cline { 2 - 4 } & $41-50$ & 27 & 26,0 \\
\cline { 2 - 4 } & $51-60$ & 31 & 29,8 \\
\cline { 2 - 4 } & 61 y más & 13 & 12,5 \\
\hline \multirow{3}{*}{ Tipo de clínica } & Adulto-pregrado & 61 & 58,7 \\
\cline { 2 - 4 } & Adulto-posgrado & 43 & 41,3 \\
\hline
\end{tabular}

Fuente: elaboración propia

En términos generales (tabla 2), las condiciones sistémicas más prevalentes fueron: enfermedad ocular (no especificada), (58\% según encuesta y $30 \%$ según la $\mathrm{HC}$, con diferencias significativas $\mathrm{p}<0,001)$ y las reacciones alérgicas ( $21 \%$ según HC y $18 \%$ según encuesta

Tabla 2. Prevalencia de enfermedades sistémicas de la población estudiada según sexo y valores totales

\begin{tabular}{|c|c|c|c|c|c|c|c|c|c|}
\hline \multirow{2}{*}{ Tipo de diagnóstico } & \multicolumn{2}{|c|}{ Hombres } & \multirow{2}{*}{$P^{c}$} & \multicolumn{2}{|c|}{ Mujeres } & \multirow{2}{*}{$P^{c}$} & \multicolumn{2}{|c|}{ Total } & \multirow{2}{*}{$P^{c}$} \\
\hline & Prev1 $^{a}$ & Prev2 $2^{b}$ & & Prev1 $^{a}$ & Prev2b & & Prev1 $^{a}$ & $\operatorname{Prev}^{b}$ & \\
\hline Migraña & 3,4 & 3,4 & 0,4718 & 9,3 & 13,3 & 0,6065 & 7,7 & 10,6 & 0,6303 \\
\hline Hipertensión arterial & 17,2 & 24,1 & 0,7458 & 17,3 & 20,0 & 0,8640 & 17,3 & 21,2 & 0,5976 \\
\hline Hipotiroidismo & 3,4 & 3,4 & 0,4718 & 6,7 & 9,3 & 0,7634 & 5,8 & 7,7 & 0,7820 \\
\hline Asma & 3,4 & 3,4 & 0,4718 & 5,3 & 5,3 & 0,7163 & 4,8 & 4,8 & 0,7459 \\
\hline Reacciones alérgicas & 13,8 & 13,8 & 0,7034 & 24,0 & 20,0 & 0,6934 & 21,2 & 18,3 & 0,7274 \\
\hline Diabetes & 6,9 & 13,8 & 0,6664 & 2,7 & 6,7 & 0,4388 & 3,8 & 8,7 & 0,2519 \\
\hline Hipertiroidismo & 0,0 & 3,4 & 1,0000 & 4,0 & 5,3 & 1,0000 & 2,9 & 4,8 & 0,7184 \\
\hline Gastritis & 3,4 & 13,8 & 0,3494 & 22,7 & 28,0 & 0,5733 & 17,3 & 24,0 & 0,3043 \\
\hline Enfermedad ocular & 34,5 & 58,6 & 0,1142 & 28,0 & 57,3 & 0,0005 & 29,8 & 57,7 & 0,0001 \\
\hline Dislipidemias & 0,0 & 20,7 & 0,0311 & 17,3 & 25,3 & 0,3190 & 12,5 & 24,0 & 0,0484 \\
\hline
\end{tabular}

a. Prevalencia tomada según historia clínica. b. Prevalencia tomada según encuesta telefónica (ЕT). c. Chi cuadrado de diferencia de proporciones. Fuente: elaboración propia 
y sin diferencias significativas). Las condiciones menos prevalentes fueron el hipertiroidismo (3\% según HC y $5 \%$ según encuesta, sin diferencias significativas) y la diabetes (sin clasificación) (4\% según HC y $9 \%$ según encuesta, sin diferencias significativas). En casi todos los casos varían las prevalencias reportadas por HC y la encuesta (con mayor reporte en la encuesta), exceptuando el asma (no hay variación) y las reacciones alérgicas (mayor reporte en la $\mathrm{HC}$ ).

Al analizar los datos desagregados por sexo (tabla 2), la enfermedad ocular es la de mayor prevalencia para ambos sexos; seguida por la hipertensión arterial en los hombres (17\% según HC y $24 \%$ según encuesta, sin diferencias significativas) y las reacciones alérgicas en las mujeres (24\% según HC y $20 \%$ según encuesta, sin diferencias significativas). Se encontraron diferencias significativas en las prevalencias reportadas para el caso de las dislipidemias en hombres ( $p<0,05$ y mayor prevalencia según encuesta) y la enfermedad ocular en las mujeres ( $p<0,001$ y mayor prevalencia según encuesta).

Según el intervalo de edad (tabla 3), la enfermedad ocular (con diferencias significativas entre el reporte por HC y encuesta) y la hipertensión arterial son las condiciones con mayor prevalencia reportada para la población mayor o igual a 51 años, y la menos prevalente, el asma. Para la población menor o igual a 50 años, las más reportadas fueron la enfermedad ocular (con diferencias significativas entre el reporte por HC y encuesta) y las reacciones alérgicas, y la de menor prevalencia, el hipertiroidismo.

Al desagregar por tipo de clínica (tabla 4), la enfermedad ocular continuó siendo la más reportada en ambas clínicas; las menos prevalentes en pregrado fue-

Tabla 3. Prevalencia de enfermedades sistémicas de la población estudiada según intervalo de edad

\begin{tabular}{|c|c|c|c|c|c|c|}
\hline \multirow{2}{*}{ Tipo de diagnóstico } & \multicolumn{2}{|c|}{$\leq 50$ años } & \multirow{2}{*}{$P^{c}$} & \multicolumn{2}{|c|}{$\geq 51$ años } & \multirow{2}{*}{$P^{c}$} \\
\hline & Prev1 $^{\mathrm{a}}$ & Prev2 ${ }^{b}$ & & Prev1 $^{\mathrm{a}}$ & Prev $2^{b}$ & \\
\hline Migraña & 8,3 & 13,3 & 0,5569 & 6,8 & 6,8 & 0,6724 \\
\hline Hipertensión arterial & 10,0 & 11,7 & 1,0000 & 27,3 & 34,1 & 0,6439 \\
\hline Hipotiroidismo & 5,0 & 6,7 & 1,0000 & 6,8 & 9,1 & 1,0000 \\
\hline Asma & 1,7 & 3,3 & 1,0000 & 9,1 & 6,8 & 1,0000 \\
\hline Reacciones alérgicas & 23,3 & 21,7 & 1,0000 & 18,2 & 13,6 & 0,7707 \\
\hline Diabetes & 0,0 & 6,7 & 0,1271 & 9,1 & 11,4 & 1,0000 \\
\hline Hipertiroidismo & 1,7 & 5,0 & 0,6110 & 4,5 & 4,5 & 0,6088 \\
\hline Gastritis & 21,7 & 21,7 & 0,8246 & 11,4 & 27,3 & 0,1052 \\
\hline Enfermedad ocular & 26,7 & 43,3 & 0,0850 & 34,1 & 77,3 & 0,0001 \\
\hline Dislipidemias & 6,7 & 11,7 & 0,5269 & 20,5 & 40,9 & 0,0644 \\
\hline
\end{tabular}

a. Prevalencia tomada según historia clínica. b. Prevalencia tomada según encuesta telefónica (ЕT). c. Chi cuadrado de diferencia de proporciones. Fuente: elaboración propia

Tabla 4. Prevalencia de enfermedades sistémicas de la población estudiada según tipo de clínica

\begin{tabular}{|c|c|c|c|c|c|c|}
\hline \multirow{2}{*}{ Tipo de diagnóstico } & \multicolumn{2}{|c|}{ Pregrado } & \multirow{2}{*}{$P^{c}$} & \multicolumn{2}{|c|}{ Posgrado } & \multirow{2}{*}{$P^{c}$} \\
\hline & Prev1 $^{a}$ & Prev $2^{b}$ & & Prev1 $^{a}$ & Prev2 ${ }^{b}$ & \\
\hline Migraña & 9,8 & 11,5 & 1,0000 & 4,7 & 9,3 & 0,6721 \\
\hline Hipertensión arterial & 18,0 & 26,2 & 0,3830 & 16,3 & 14,0 & 1,0000 \\
\hline Hipotiroidismo & 8,2 & 11,5 & 0,7611 & 2,3 & 2,3 & 0,4743 \\
\hline Asma & 6,6 & 6,6 & 0,7146 & 2,3 & 2,3 & 0,4743 \\
\hline Reacciones alérgicas & 19,7 & 14,8 & 0,6315 & 23,3 & 23,3 & 0,7985 \\
\hline Diabetes & 3,3 & 11,5 & 0,1659 & 4,7 & 4,7 & 0,6086 \\
\hline Hipertiroidismo & 3,3 & 6,6 & 0,6755 & 2,3 & 2,3 & 0,4743 \\
\hline Gastritis & 14,8 & 21,3 & 0,4799 & 20,9 & 27,9 & 0,6157 \\
\hline Enfermedad ocular & 29,5 & 55,7 & 0,0060 & 30,2 & 60,5 & 0,0093 \\
\hline Dislipidemias & 14,8 & 29,5 & 0,0810 & 9,3 & 16,3 & 0,5185 \\
\hline
\end{tabular}

a. Prevalencia tomada según historia clínica. b. Prevalencia tomada según encuesta telefónica (вт). c. Chi cuadrado de diferencia de proporciones. Fuente: elaboración propia 
ron diabetes (3\% según HC y $12 \%$ según encuesta, sin diferencias significativas) e hipertiroidismo (3\% según HC y 7\% según encuesta), y en posgrado asma, hipotiroidismo e hipertiroidismo en iguales porcentajes (3\% HC y $3 \%$ según encuesta).

Analizando los niveles de concordancia generales (tabla 5), la enfermedad con mayor concordancia en los reportes, tanto por HC como por encuesta, se dio para el asma, con un $88 \%$, y para la hipertensión arterial con un $84 \%$. Las concordancias más débiles fueron para el hipertiroidismo (22\%) y para enfermedad ocular (37\%). Según sexo (tabla 5), para el caso de los hombres se presentaron altos niveles de concordancia para migraña, hipotiroidismo, asma y reacciones alérgicas ( $100 \%$ en todos los casos), y el nivel más bajo se observó para gastritis (37\%). En cuanto a las mujeres, la concordancia más alta se observó para el caso de la hipertensión arterial, con un $86 \%$, y la más débil en la diabetes (32\%).

Según intervalo de edad (tabla 6), el mayor porcentaje de concordancia entre la población menor o igual a 50 años fue de hipotiroidismo (85\%) y el menor porcentaje de concordancia fue de hipertiroidismo (-3\%); el mayor porcentaje de concordancia en la población mayor o igual a 51 años fue el asma (100\%) y el menor fue de migraña (28\%). Por último, atendiendo al tipo de clínica, el nivel de concordancia más alto en la clínica de pregrado la presentó el asma (100\%) y el hipotiroidismo (78\%). El hipertiroidismo y la enfermedad ocular tuvieron bajas correlaciones (30 y $37 \%$, respectivamente).

Tabla 5. Concordancia diagnóstica de enfermedades sistémicas en la población estudiada según sexo y valores totales

\begin{tabular}{|l|c|c|l|c|c|l|l|l|l|}
\hline \multirow{2}{*}{ Tipo de diagnóstico } & \multicolumn{3}{|c|}{ Hombres } & \multicolumn{3}{c|}{ Mujeres } & \multicolumn{3}{c|}{ Total } \\
\cline { 2 - 11 } & Kappa & IC $_{95 \%}$ & $\begin{array}{c}\text { Fuerza de la } \\
\text { concordancia }\end{array}$ & Kappa & IC $_{95 \%}$ & $\begin{array}{c}\text { Fuerza de la } \\
\text { concordancia }\end{array}$ & Kappa & \multicolumn{1}{|c|}{ IC $_{95 \%}$} & $\begin{array}{c}\text { Fuerza de la } \\
\text { concordancia }\end{array}$ \\
\hline Migraña & 1,000 & $1,000-1,000$ & Muy buena & 0,534 & $0,207-0,862$ & Moderada & 0,594 & $0,304-0,884$ & Moderada \\
\hline Hipertensión arterial & 0,631 & $0,293-0,968$ & Buena & 0,863 & $0,712-1,015$ & Muy buena & 0,841 & $0,706-0,977$ & Muy buena \\
\hline Hipotiroidismo & 1,000 & $1,000-1,000$ & Muy buena & 0,784 & $0,490-1,079$ & Buena & 0,822 & $0,579-1,066$ & Muy buena \\
\hline Asma & 1,000 & $1,000-1,000$ & Muy buena & 0,850 & $0,558-1,142$ & Muy buena & 0,884 & $0,657-1,110$ & Muy buena \\
\hline Reacciones alérgicas & 1,000 & $1,000-1,000$ & Muy buena & 0,651 & $0,437-0,865$ & Buena & 0,683 & $0,556-0,897$ & Buena \\
\hline Diabetes & 0,630 & $0,137-1,123$ & Buena & 0,318 & $-0,332-0,968$ & Débil & 0,476 & $0,070-0,882$ & Moderada \\
\hline Hipertiroidismo & -- & -- & No aplicable & 0,251 & $-0,384-0,885$ & Débil & 0,221 & $-0,383-0,826$ & Débil \\
\hline Gastritis & 0,365 & $-0,347-1,045$ & Débil & 0,647 & $0,444-0,851$ & Buena & 0,621 & $0,428-0,813$ & Buena \\
\hline Enfermedad ocular & 0,411 & $0,091-0,730$ & Moderada & 0,349 & $0,146-0,551$ & Débil & 0,366 & $0,195-0,537$ & Débil \\
\hline Dislipidemias & -- & -- & No aplicable & 0,605 & $0,378-0,833$ & Buena & 0,495 & $0,268-0,722$ & Moderada \\
\hline
\end{tabular}

Fuente: elaboración propia

Tabla 6. Concordancia diagnóstica de enfermedades sistémicas en la población estudiada según intervalo de edad

\begin{tabular}{|l|c|c|l|c|c|l|}
\hline \multirow{2}{*}{ Tipo de diagnóstico } & \multicolumn{3}{|c|}{$\leq \mathbf{5 0}$ años } & \multicolumn{3}{c|}{$\geq \mathbf{5 1}$ años } \\
\cline { 2 - 7 } & Kappa & IC $_{95 \%}$ & $\begin{array}{c}\text { Fuerza de la } \\
\text { concordancia }\end{array}$ & Kappa & IC $_{95 \%}$ & $\begin{array}{c}\text { Fuerza de la } \\
\text { concordancia }\end{array}$ \\
\hline Migraña & 0,741 & $0,456-1,025$ & Buena & 0,283 & $-0,386-0,952$ & Débil \\
\hline Hipertensión arterial & 0,814 & $0,562-1,067$ & Muy buena & 0,839 & $0,663-1,015$ & Muy buena \\
\hline Hipotiroidismo & 0,848 & $0,552-1,143$ & Muy buena & 0,787 & $0,375-1,199$ & Buena \\
\hline Asma & 0,659 & $-0,004-1,322$ & Buena & 1,000 & $1,000-1,000$ & Muy buena \\
\hline Reacciones alérgicas & 0,761 & $0,561-0,962$ & Buena & 0,662 & $0,345-0,978$ & Buena \\
\hline Diabetes & -- & -- & No aplicable & 0,725 & $0,353-1,097$ & Buena \\
\hline Hipertiroidismo & $-0,026$ & $-0,997-0,945$ & Pobre & 0,474 & $-0,236-1,185$ & Moderada \\
\hline Gastritis & 0,803 & $0,616-0,989$ & Muy buena & 0,369 & $0,002-0,737$ & Débil \\
\hline Enfermedad ocular & 0,573 & $0,358-0,789$ & Moderada & 0,109 & $-0,142-0,361$ & Pobre \\
\hline Dislipidemias & 0,503 & $0,086-0,920$ & Moderada & 0,435 & $0,147-0,723$ & Moderada \\
\hline
\end{tabular}


Finalmente, según tipo de clínica (tabla 7), en la clínica de posgrado la hipertensión arterial (100\%), hipotiroidismo (100\%) y la gastritis (81\%) tuvieron la más alta correlación, y los niveles más débiles de correlación se presentaron en los casos de migraña (29\%), hipertiroidismo (-2\%) y enfermedad ocular (36\%). a los estudiantes por parte de los docentes de la clínica en la correlación y aplicación de los conceptos teóricos.

La enfermedad que presentó mayor nivel de concordancia fue el asma, lo cual podría explicarse debido a que los signos y síntomas son muy evidentes para el paciente y su tratamiento es muy delimitado, lo que

Tabla 7. Concordancia diagnóstica de enfermedades sistémicas en la población estudiada según tipo de clínica

\begin{tabular}{|l|c|c|l|c|c|l|}
\hline \multirow{2}{*}{ Tipo de diagnóstico } & \multicolumn{3}{|c|}{ Pregrado } & \multicolumn{3}{c|}{ Posgrado } \\
\cline { 2 - 8 } & Kappa & IC $_{95 \%}$ & $\begin{array}{c}\text { Fuerza de la } \\
\text { concordancia }\end{array}$ & Kappa & IC $_{95 \%}$ & $\begin{array}{c}\text { Fuerza de la } \\
\text { concordancia }\end{array}$ \\
\hline Migraña & 0,740 & $0,454-1,026$ & Buena & 0,288 & $-0,386-0,952$ & Débil \\
\hline Hipertensión arterial & 0,761 & $0,561-0,961$ & Buena & 1,000 & $1,000-1,000$ & Muy buena \\
\hline Hipotiroidismo & 0,780 & $0,482-1,079$ & Buena & 1,000 & $1,000-1,000$ & Muy buena \\
\hline Asma & 1,000 & $1,000-1,000$ & Muy buena & --- & --- & No aplicable \\
\hline Reacciones alérgicas & 0,714 & $0,473-0,954$ & Buena & 0,739 & $0,496-0,983$ & Buena \\
\hline Diabetes & 0,414 & $-0,078-0,905$ & Moderada & 0,655 & $-0,012-1,323$ & Buena \\
\hline Hipertiroidismo & 0,301 & $-0,360-0,962$ & Débil & $-0,024$ & $-1,409-1,362$ & Débil \\
\hline Gastritis & 0,448 & $0,135-0,760$ & Moderada & 0,812 & $0,607-1,017$ & Muy buena \\
\hline Enfermedad ocular & 0,374 & $0,149-0,599$ & Débil & 0,356 & $0,093-0,619$ & Débil \\
\hline Dislipidemias & 0,401 & $0,112-0,690$ & Moderada & 0,690 & $0,351-1,028$ & Buena \\
\hline
\end{tabular}

Fuente: elaboración propia

\section{Discusión}

Los hallazgos principales de esta investigación dan cuenta de la concordancia en el reporte de algunas de las condiciones sistémicas de mayor prevalencia. La condición sistémica más prevalente fue la enfermedad ocular, y la menos prevalente, el hipertiroidismo. El análisis kappa mostró que las concordancias más débiles se presentaron para el hipertiroidismo y para la enfermedad ocular. Hubo variaciones en las prevalencias y en los índices de concordancia de acuerdo con el sexo, la clínica y el intervalo de edad.

Las diferencias halladas por los análisis de prevalencia y concordancia entre la información consignada en la HC y la obtenida por ET, tanto en la clínica del adulto de pregrado como de posgrado y cuyo producto final es el subregistro, podrían tener varias causas: a) dificultades en la comunicación y, por ende, en el registro de la información del clínico con el paciente al realizar la entrevista de primera vez; b) desconocimiento de los estudiantes de la importancia e implicaciones que tienen algunas condiciones sistémicas para el odontólogo en sus procedimientos; c) instrumentos (HC) poco adecuados que no permiten un diligenciamiento ágil y óptimo por parte de los estudiantes; d) poca exigencia hace que sea fácilmente reconocida [13, 14]. El segundo más común fue la hipertensión arterial, y esto podría explicarse porque es un diagnóstico muy común en nuestra población, y en el Sistema de Salud vigente se cuenta con programas de promoción y prevención que incluyen esta condición, y por tanto los pacientes se apropian de su condición sistémica $[15,16]$.

En relación con la interpretación de los resultados, es importante comentar las fortalezas y limitaciones de esta investigación. La inclusión de pacientes atendidos en la Facultad permitió un fácil acceso a la información de las HC, constituyéndose en una fortaleza dentro del estudio, además de los instrumentos de recolección de información que fueron cuidadosamente diseñados y evaluados mediante consulta por expertos, prueba piloto y concordancia en el registro entre las observadoras. La diferencia en el tiempo de recolección de la información por vía telefónica y la obtenida en las historias clínicas representan una limitación, ya que en la mayoría hay un lapso de por lo menos seis meses, tiempo en el cual se pudo haber diagnosticado una nueva condición, lo que podría alterar ligeramente los resultados. Si bien estos son aplicables al contexto de la Institución, deben interpretarse con cautela puesto que el muestreo por conveniencia no permite hacer 
generalizaciones; en algunos casos no se pudo obtener más muestras porque muchos pacientes no accedieron a participar en el estudio y otros no pudieron ser contactados. No obstante, dado que la Facultad de Odontología es una institución formadora de talento humano en salud bucal, en la cual se atienden pacientes de todas las zonas de Medellín, este estudio piloto se constituye en una propuesta para otras investigaciones con muestreos representativos.

\section{Conclusiones}

Teniendo en cuenta los resultados obtenidos en este estudio, se hace indispensable el mejoramiento de la calidad de los registros consignados en las HC para favorecer la creación de sistemas de vigilancia epidemiológica de las principales condiciones sistémicas de los pacientes atendidos en la Facultad, el diseño de guías de atención específicos para la atención odontológica de dichos pacientes con cada entidad y el favorecimiento de futuros estudios en esta línea de investigación. Igualmente, es preciso exigir al odontólogo y especialista en formación la integración en el escenario clínico de todos los conocimientos teóricos de semiología médica, procurando la adecuada apropiación de estos, para así propiciar el manejo integral del paciente.

\section{Referencias}

[1] Díaz 1, Castellanos J, Gay O. Medicina en odontología, manejo dental de pacientes con enfermedades sistémicas. 2a ed. México: El Manual Moderno; 2002.

[2] Castaño A, Roldán J. Manual de introducción a la odontología. Madrid: Médica Ripano; 2005.

[3] Consejo General de Colegios Oficiales de Odontólogos y Estomatólogos de España. Protocolos clínicos aceptados por el Ilustre Consejo General de Colegios Oficiales de Odontólogos y Estomatólogos de España. [Internet]. [Consultado: 8 de febrero de 2011]. Disponible en: http://www.consejodentistas.es/pdf/prevencion.pdf

[4] Little J, Falace D, Miller C, Rhodus N. Tratamiento odontológico del paciente bajo tratamiento médico. $5 .^{\mathrm{a}}$ ed. Madrid: Harcourt Brace; 1998.

[5] González M. La historia clínica como instrumento de método clínico y su importancia en la realización del tratamiento odontológico. Caracas: Universidad Central de Venezuela; 1994.

[6] Alfonso A, Moreno O, Hernández MN, Pardo R, Alfonzo T. Prevalencia de pacientes sistémicamente compro- metidos que acuden al CIDC (Centro de Investigación y Diagnóstico Clínico). III Congreso de la Región Latinoamericana; International Association for Dental Research (IADR) y VIII de la División Venezolana de la IAD; 2009 Isla Margarita- Venezuela; Nov 11-13. [Internet] [Consultado: 8 de febrero de 2011] Disponible en: http://iadr.confex.com/iadr/venez09/preliminaryprogram/abstract_126198.htm

[7] La Mora-Franz E de, Carrillo-Tamez ME. Systemic disease in the Pediatric Dental Department during 1984. Rev ADM. 1990; 47(3): 97-102.

[8] Dumitrescu AL. Occurrence of self-reported systemic medical conditions in patients with periodontal disease. Rom J Intern Med. 2006; 44(1): 35-48.

[9] Antioquia. Gobernación. Secretaría Seccional de Salud y Protección Social. Estadísticas de Morbilidad. [Internet] [Consultado: 4 de mayo de 2012]. Disponible en: http://www.dssa.gov.co/index.php/estadisticas/morbilidad

[10] López de Ullibarri Galparsoro I, Pita Fernández S. Medidas de concordancia: el índice Kappa. Cad Aten Primaria. 1999; 6: 169-71.

[11] Landis JR, Koch GG. The measurement of observer agreement for categorical data. Biometrics.1977; 33: 159-74.

[12] Colombia. Ministerio de Salud y Protección Social. Resolución no 008430 de 1993 (4 de octubre de 1993). Por la cual se establecen las normas científicas, técnicas y administrativas para la investigación en salud. Bogotá: El Ministerio; 1993.

[13] Castillo Laita JA, De Benito Fernández J, Escribano Montaner A, Fernández Benítez M, García de la Rubia $\mathrm{S}$, Garde Garde J et al. Consenso sobre el tratamiento del asma en pediatría. An Pediatr (Barc). 2007; 67(3): 253-73.

[14] Salas Hernández J, Ramírez Venegas A, Carrillo Rodríguez G, Pérez Chavira R, Sansores Martínez RH, Mejía Ávila $\mathrm{M}$ et al. La conducta de un grupo de médicos mexicanos ante el diagnóstico y tratamiento del asma. Rev Inst Nac Enfermedades Respir. 1994; 7(2): 116-21.

[15] Marín R de la Sierra A, Armario P, Campos C, Banegas JR, Gorostidi M. Guía sobre el diagnóstico y tratamiento de la hipertensión arterial en España. 2005. Med Clin (Barc). 2005; 125(1): 24-34.

[16] Colombia. Ministerio de la Protección Social. Estudio de ajuste de la UPC-S por la inclusión en el POS-S de actividades para el manejo de hipertensión arterial y diabetes mellitus tipo 2 en personas de 45 años o más. 2008. [Internet] [Consultado: 22 de mayo de 2012]. Disponible en: http://www.pos.gov.co/Documents/HTA-DM_POSS_ABRIL_08_ULTIMA\%20 VERSI\%C3\%93N.pdf 